Trauma Berufskrankh 2012 • 14[Suppl 3]:317-323 DOI 10.1007/s10039-011-1825-x

Online publiziert: 22. März 2012

(c) Springer-Verlag 2012
H. Kohler

Abteilung für Berufsgenossenschaftliche Rehabilitation und Heilverfahrenssteuerung,

BG-Unfallklinik Ludwigshafen

\title{
Medizinisch-beruflich orientierte Rehabilitation
}

\section{Konzept und erste Ergebnisse}

\section{Impuls und Entwicklung des neuen Rehabilitations- verfahrens}

Von der medizinischen Versorgung bis zur beruflichen Reintegration ist die gesetzliche Unfallversicherung für den Patienten zuständig. Die medizinische, berufliche und soziale Rehabilitation soll mit al len geeigneten Mitteln erfolgen. Durch den demografischen Wandel und die knapper werdenden finanziellen Ressourcen werden die Rehabilitationsmaßnahmen aber auch von den Unfallversicherungsträgern zunehmend kritisch hinterfragt.

\section{Berufsanforderungen heute}

In den letzten Jahrzehnten kam es zu einer entscheidenden Veränderung klassischer Berufsbilder. Arbeitnehmer, die nach einem Arbeitsunfall wieder ins Berufsleben integriert werden sollen, müssen sich daher besonderen Herausforderungen stellen.

Der sich immer schneller wandelnde Arbeitsmarkt führt bei den Arbeitnehmern zu erheblichen physischen und psychischen Belastungen. Hierbei spielen u. a. folgende Faktoren eine Rolle:

- neue Technologien im Arbeitsalltag

- Änderungen der täglichen Arbeitszeit sowie der Lebensarbeitszeit

- Bildschirmarbeit

- Leiharbeit und Zeitverträge

- psychische Belastungen am Arbeitsplatz
Wenn die durchgeführten Rehabilitationsmaßnahmen weiterhin Wirkung zeigen sollen, müssen die veränderten Rahmenbedingungen zwingend in der Rehabilitationspraxis berücksichtigt werden $[5,8]$.

Sowohl die Verkürzung der Zeitspanne vom Beginn der Arbeitsunfähigkeit nach einem Arbeitsunfall bis zur beruflichen Reintegration als auch die Vermeidung von Verletzten- und Erwerbsunfähigkeitsrenten haben eine sehr hohe finanzielle Relevanz. Aus psychologischer Sicht ist eine lange Wartezeit zwischen dem Abschluss der medizinischen und dem Beginn der beruflichen Rehabilitation unbedingt zu vermeiden, denn diese wirkt auf die Patienten stark demotivierend. Demotivation aber reduziert das Selbstbewusstsein der Patienten, und der bereits erreichte funktionelle Status geht wieder verloren [9].

Studien ergaben, dass nicht nur der erreichte funktionelle Status eine Rolle spielt, ob der Patient wieder arbeiten kann, sondern insbesondere auch seine Motivationslage, finanzielle Erwägungen und die Einschätzung des Patienten selbst bezüglich seiner beruflichen Situation [2].

Die Beschäftigung von Arbeitnehmern mit Behinderungen auf dem ersten Arbeitsmarkt ist keine Selbstverständlichkeit, auch wenn die Pflicht hierzu ab einer gewissen Unternehmensgröße im SGB (Sozialgesetzbuch) IX gesetzlich verankert ist und das Integrationsamt die Beschäftigung von derartig benachteiligten Arbeitnehmern fördert [3]. Die Arbeit- geber sind zurückhaltend, da Arbeitnehmern mit Behinderungen Privilegien wie ein besonderer Kündigungsschutz und zusätzliche Urlaubstage eingeräumt werden. Sie befürchten u. a. eine Mehrbelastung der anderen Kollegen durch erhöhte Arbeitsausfälle sowie teure Umstrukturierungsmaßnahmen der Arbeitsplätze und -abläufe. Aus diesem Grund ist es besonders wichtig, dass Arbeitnehmer mit Behinderungen berufsspezifisch auf ihren Arbeitseinsatz vorbereitet werden.

\section{Berufsbezogene Rehabilitation}

Die Kostenträger verlangen seit Jahren, im Rahmen der Rehabilitation verstärkt berufsbezogene therapeutische Maßnahmen anzubieten $[1,10]$. Bisher wurde dieser Aspekt in keinem Rehabilitationsverfahren berücksichtigt. Zwar versuchten vereinzelte private Rehabilitationsdienstleister, diese Ansätze zu beachten, jedoch nur in geringem Ausmaß und ohne dass ein medizinisches Konzept zugrunde lag. In der BG-Unfallklinik (BG: Berufsgenossenschaft) Ludwigshafen wurde deshalb beschlossen, das Leistungsspektrum mit einem einzigartigen, berufsspezifischen Konzept zu erweitern.

Im Jahr 2007 wurde zunächst die Abteilung für berufsgenossenschaftliche Rehabilitation und Heilverfahrenssteuerung an der BG-Unfallklinik Ludwigshafen gegründet. Sie wurde mit der Durchführung aller ambulanten und stationären Rehabilitationsmaßnahmen, einschließlich der gesamten BG-Ambulanz, an der 


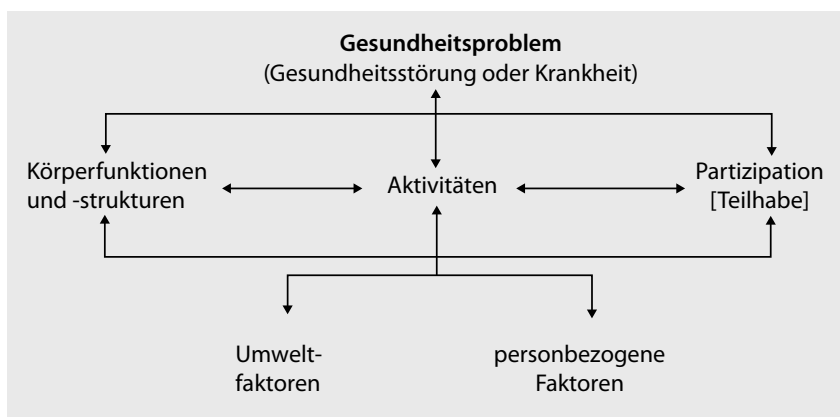

Abb. 14 Mögliche Zusammenhänge und Interaktionen zwischen Gesundheitsproblem (Krankheit/Unfall) und den Auswirkungen auf Aktivitäten und Teilhabe sowie die Einflussnahme von Kontextfaktoren auf die Rehabilitation der Patienten (Modell der WHO)
Klinik beauftragt. Hierdurch sollten eine verbesserte Anbindung und Kommunikation mit den Unfallversicherungsträgern erreicht werden. Weiteres Ziel war die optimierte Berücksichtigung der Kundenwünsche.

Der Träger der BG-Unfallklinik Ludwigshafen erteilte den Auftrag, ein berufsspezifisches Rehabilitationsverfahren unter Berücksichtigung der spezifischen Rahmenbedingungen des berufsgenossenschaftlichen Heilverfahrens zu entwickeln. Das Ergebnis ist die medizinischberuflich orientierte (MBO) Rehabilitation an der BG-Unfallklinik Ludwigshafen. Die Besonderheit bei deren Entwicklung war eine interdisziplinäre Arbeitsgruppe, in der alle am MBO-Rehabilitationsverfahren beteiligten Berufsgruppen einschließlich der Kostenträger vertreten waren. Diese Arbeitsgruppe stimmte das Konzept der MBO-Rehabilitation auf die Bedürfnisse des doppelten Kunden im Gesundheitswesen ab, d. h. sowohl die Interessen der Patienten als auch die der Kostenträger wurden berücksichtigt. $\mathrm{Da}-$ mit wurde der Tatsache Rechnung getragen, dass Kunde im Gesundheitswesen nicht nur der Patient, sondern auch der Kostenträger ist.

Nach einer Entwicklungsphase von $1 \mathrm{Jahr}$ startete die medizinisch-beruflich orientierte Rehabilitation in ihre Testphase. Zur ihrer Einführung am Markt fand am 25.11.2008 ein großes Symposium in der BG-Unfallklinik Ludwigshafen statt, bei dem sich die Teilnehmer persönlich ein Bild vom Leistungsangebot der MBORehabilitation machen konnten. Die Resonanz auf dieses Symposium war überwältigend und hatte zur Folge, dass die Nachfrage nach diesen speziellen Therapieplätzen von Anfang an groß war und weitere Therapieplätze eingerichtet werden konnten. Durch Kooperation mit ex- ternen Partnern konnte auch das Leistungsangebot weiter ausgebaut werden.

Die Mitarbeiter der Bereiche Physiound Ergotherapie wurden vorab geschult, insbesondere in den Bereichen EFL (Evaluation der funktionellen Leistungsfähigkeit nach Isernhagen) und ICF (internationale Klassifikation der Funktionsfähigkeit, Behinderung und Gesundheit).

\section{Wissenschaftliche Basis, Klassifikationssysteme}

Die medizinische Rehabilitation basiert auf theoretisch fundierten und wissenschaftlich ausgewiesenen Konzepten. Für die theoretische Einordnung und das wissenschaftliche Therapiekonzept der MBO steht das WHO-Modell (WHO: Weltgesundheitsorganisation; - Abb. 1) der ICF. Zur Bestimmung des Rehabilitationsbedarfs und zur Messung des Rehabilitationserfolgs kann ebenfalls die ICF herangezogen werden. Außerdem liegen die aus diesem Modell abgeleiteten Behandlungsmaßnahmen und -elemente als standardisierte Leistungsbeschreibung vor [12].

Die medizinisch-beruflich orientierte Rehabilitation berücksichtigt in besonderem Maße die individuelle berufliche Problemlage. Dies erwies sich nicht nur in Bezug auf die Leistung und Leistungsfähigkeit der Patienten, sondern auch in Bezug auf Teilhabe und Kosteneffektivität als wirksam [4, 7]. Auch das Risiko der Arbeitslosigkeit kann durch die MBO-Rehabilitation signifikant reduziert werden [11]. Über den orthopädischen Indikationsbereich hinaus wurden erste positive Erfahrungen einer MBO-Rehabilitation bei onkologischen Patienten berichtet [6]

Zur Beschreibung der psychosozialen Zusammenhänge eines Gesundheitsproblems bedarf es eines biopsychosozialen Ansatzes, der die theoretische Grundlage der ICF bildet. Klassifikationen sind naturgemäß vorwiegend statische Beschreibungen oder Bezeichnungen eines $\mathrm{Zu}$ stands, eines Syndroms oder einer Krankheit [z. B. ICD-Schlüssel (ICD: internationale Klassifikation der Krankheiten)]. Die ICF bemüht sich jedoch, neben der Beschreibung von Körper-/Organstrukturen und Körper-/Organfunktionen um die Einbeziehung von sog. Kontextfaktoren, welche die Funktionsfähigkeit einer Person mit beeinflussen und damit Funktionsstörungen verstärken oder abschwächen. Weiterhin ermöglicht die ICF, die Zusammenhänge zwischen Schädigung und Funktionsstörung einerseits und Alltagsaktivitäten andererseits zu erfassen und damit auch die vom Gesetzgeber geschützte Teilhabe (gesellschaftliche Integration, privat und beruflich) darzustellen und zu beurteilen.

Der auf dieser Basis entstandene Begriff der Funktionsfähigkeit umfasst alle Aspekte der funktionalen Gesundheit. Eine Person ist funktional gesund, wenn

- ihre körperlichen Funktionen und -strukturen denen eines gesunden Menschen entsprechen,

- sie all das tun kann, was von einem Menschen ohne Gesundheitsproblem erwartet wird, und

- sie ihr Dasein in allen Lebensbereichen, die für sie wichtig sind, entfalten kann wie ein Mensch ohne gesundheitsbedingte Beeinträchtigung.

Kontextfaktoren stellen den gesamten Lebenshintergrund eines Menschen dar. Sie umfassen jedoch insbesondere 2 Komponenten:

- die Umweltfaktoren und

- die personenbezogenen Faktoren.

Die Kontextfaktoren haben Einfluss auf den Menschen mit oder ohne Erkrankung. Sie beeinflussen die Krankheitsentstehung (Risiko und Risikobereitschaft) und das Ausmaß von Funktionsstörungen und deren Bedeutung für die weitere Krankheitsentwicklung und Rehabilitation. Die Kontextfaktoren als Mitverursacher einer Erkrankung oder eines Unfalls einerseits und als Einflussfaktoren auf die Entwicklung und Bedeutung einer Funktionsstörung andererseits machen aber auch deutlich, dass das biomedizinische 
und das biopsychosoziale Modell einander ergänzen und nur ihre gemeinsame Betrachtung und Berücksichtigung dem Anspruch einer ganzheitlichen Rehabilitation nahe kommen (• Abb. 1). Diese gemeinsame Betrachtungsweise soll deshalb auch als Grundlage der Maßnahmen im Rahmen der MBO-Rehabilitation dienen.

Wichtig bei der Evaluation und Rehabilitation der Patienten ist der Umstand, dass die Kontextfaktoren (Umweltfaktoren, personenbezogene Faktoren) nicht nur Einflussfaktoren der Unfallfolgen, sondern selbst auch primäre Unfallursache sein können. Ihre Berücksichtigung ist somit nicht nur für den Heilungserfolg, sondern auch für die Primär- und Sekundärprävention von Unfällen entscheidend. Unter Beachtung der im klinischen Alltag erfahrbaren Kette von Unfallfolgen bis hin zur Störung der Teilhabe - deren Wiederherstellung der primäre gesetzliche Auftrag an die Rehabilitation darstellt - kann das obige Schema etwas modifiziert werden ( $\bullet$ Abb. 2). In dieser Modifikation kann auch die Aufgabenverteilung von Chirurgie/Akutmedizin und Rehabilitation verdeutlicht werden.

Die ICF bildet als Klassifikationssystem eine wichtige Grundlage in der Rehabilitation unfallverletzter Patienten, da sie neben der Beschreibung und Klassifizierung von Schädigungen bzw. einer Beeinträchtigung einer Körperfunktion oder -struktur die Beurteilung der damit im Zusammenhang stehenden Beeinträchtigungen von Aktivitäten und Teilhabe erlaubt und darüber hinaus die fördernden oder hemmenden Kontextfaktoren mit einbezieht. Funktionsstörungen und Einschränkungen der Aktivitäten können direkt mit den individuellen beruflichen Anforderungen in Bezug gesetzt werden. Daher ist die ICF bei der Interventionsplanung und der Evaluation rehabilitativer Maßnahmen einzusetzen. Entsprechend gezielt können die therapeutischen Maßnahmen insbesondere in der MBORehabilitation durchgeführt werden. Die ICF bietet somit eine geeignete Grundlage, Therapieerfolge nach einem international anerkannten Modell zu beschreiben und $\mathrm{zu}$ quantifizieren und in einer international anerkannten Sprache darzustellen.

Trauma Berufskrankh 2012 - 14[Suppl 3]:317-323 DOI 10.1007/s10039-011-1825-x

(c) Springer-Verlag 2012

H. Kohler

\section{Medizinisch-beruflich orientierte Rehabilitation. Konzept und erste Ergebnisse}

\section{Zusammenfassung}

Viele Patienten haben nach einem Arbeitsunfall Probleme, in ihren alten Beruf zurückzufinden, obwohl die eigentliche Verletzung ausgeheilt ist. Dabei ist gesetzlich geregelt, dass die Rehabilitation nach einem Arbeitsunfall dem Patienten - wo immer möglich den Weg zurück ins Berufsleben ermöglichen soll. In der BG-Unfallklinik (BG: Berufsgenossenschaft) Ludwigshafen wurde deshalb ein innovatives Rehabilitationskonzept entwickelt und eingeführt, das Patienten nach einem Arbeitsunfall gezielt in die Arbeitsfähigkeit zurückführt: die medizinisch-beruflich orientierte Rehabilitation. Hierunter versteht man die Wiederherstellung, Anpassung und Optimierung des individuellen Fähigkeitsprofils eines Patienten in Richtung des individuellen beruflichen Anforderungsprofils. Die Arbeitsplatzsituation wird im Rehabilitationsbereich der Klinik simuliert. Die Patienten werden durch arbeitsplatzspezifische, therapeutische Maßnahmen gezielt in Richtung Arbeitsfähigkeit trainiert. Durch die örtliche Einheit von chirurgischer und akutmedizinischer Expertise einerseits und der Rehabilitationsexpertise andererseits ist es möglich, die Patienten von der Unfallstelle bis zur medizinisch-beruflich orientierten Rehabilitation aus einer Hand zu versorgen.

\section{Schlüsselwörter}

Arbeitsunfälle · Gesetz · Rehabilitation .

Medizinisch-beruflich orientierte

Rehabilitation · Arbeitsfähigkeit

\section{Work oriented medical rehabilitation. Concept and first results}

\begin{abstract}
Many patients have problems returning to their original profession after an occupational accident despite their complete recovery. It is regulated by law that rehabilitation after an occupational accident is intended to enable-whenever possible - the patients to find their way back into working life. The BGU Ludwigshafen developed an innovative concept of rehabilitation, which targets the complete recovery of the patients' working ability. It is called work oriented medical rehabilitation. The concept deals with the recovery, adjustment, and optimization of the individual ability profile of the patient in relation to his/her individual professional requirement profile in the job. The activities at the place of work are simulated in the occupational
\end{abstract}

therapy department of the BGU. As a result, the patients are trained towards full working ability through targeted therapeutic measures that simulate specific conditions of the patients' place of employment. By combining the local unit of surgical and acute medical expertise on the one hand and the expertise of rehabilitation on the other hand it is possible to treat the patient starting from the place of injury until full work oriented medical rehabilitation.

\section{Keywords}

Occupational accidents - Law - Rehabilitation . Work oriented medical rehabilitation . Ability to work

\section{Medizinisch-beruflich orientierte Rehabilitation}

\section{Ablauf}

Im Rahmen eines 2-tägigen Assessments ermittelt ein Team aus Ärzten und Therapeuten zusammen mit dem Patienten seine individuellen Fähigkeiten und stellt fest, welche Einschränkungen er durch den Unfall erlitt. Insbesondere werden die Kontextfaktoren herausgearbeitet. Gleichzeitig wird festgestellt, welche Anforde- rungen sein Beruf gemeinhin an den $\mathrm{Pa}$ tienten stellt. Aus diesem Abgleich ergibt sich eine Art Fahrplan für den Verlauf des insgesamt 4-wöchigen medizinisch-beruflich orientierten Rehabilitationsprogramms. Das individuelle berufliche Anforderungsprofil des Patienten bestimmt dessen Inhalte.

Die MBO-Rehabilitation erstreckt sich in der Regel über einen Zeitraum von 4 Wochen. Insgesamt werden von den $\mathrm{Pa}$ tienten täglich $8 \mathrm{~h}$ Therapie abgeleistet, welche sich aus arbeitsspezifischen und 


\section{Psychische, berufliche und soziale Aspekte}

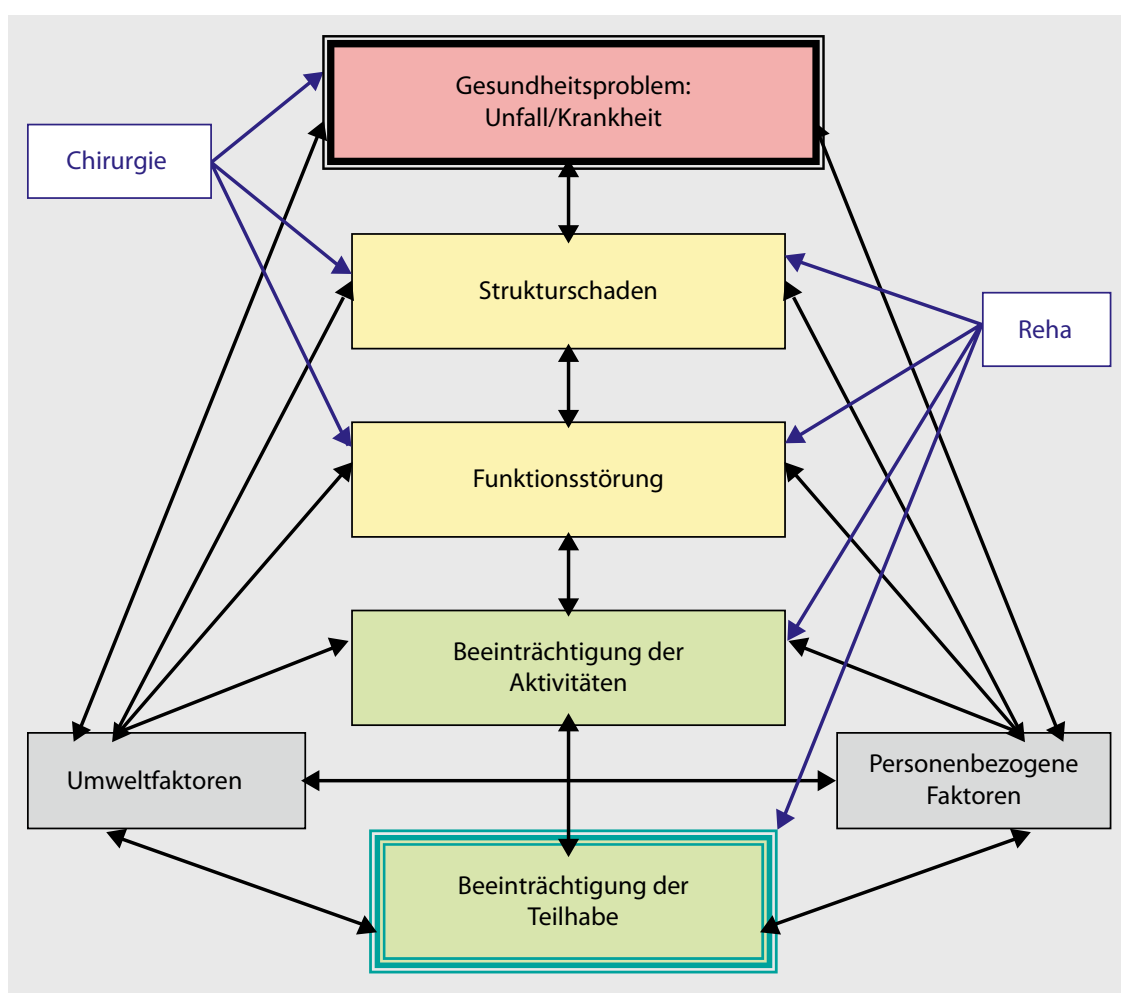

Abb. $2 \Delta$ Darstellung der allgemeinen Unfallfolgen und deren potenzieller Interaktionen und Rückwirkungen unter Berücksichtigung der Kontextfaktoren (Umweltfaktoren und personenbezogene Faktoren) sowie der therapeutischen Ebenen von Akutbehandlung/Chirurgie und Rehabilitation (Reha)

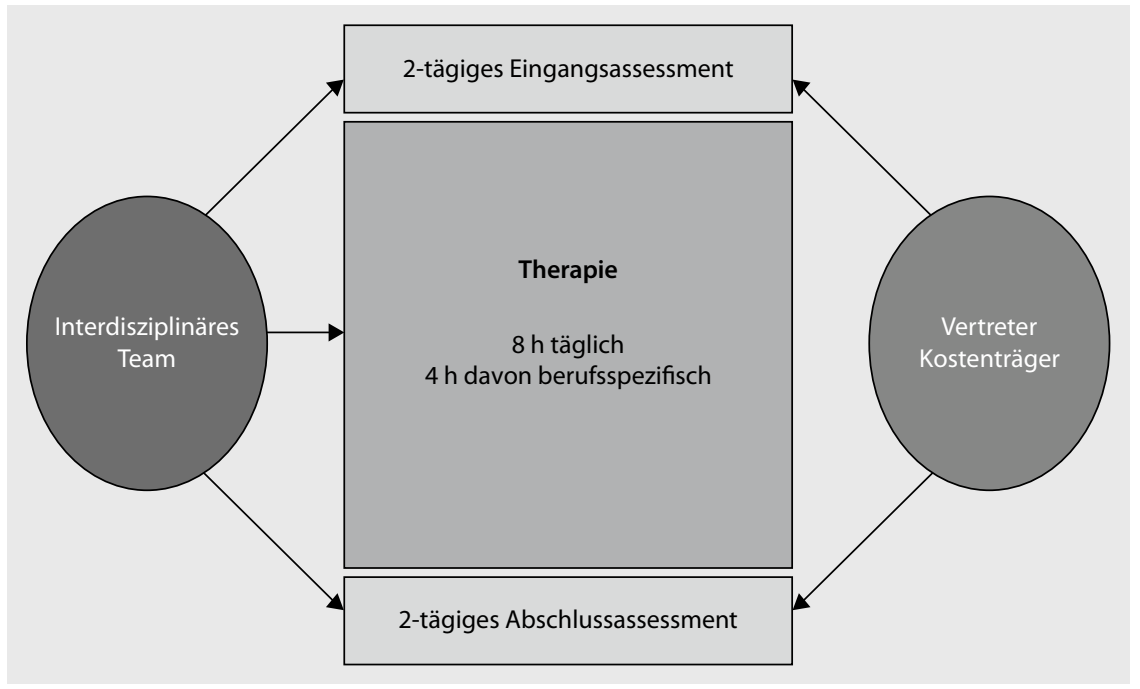

Abb. $3 \Delta$ Ablauf der MBO-Rehabilitation an der BG-Unfallklinik Ludwigshafen

konventionellen Maßnahmen zusammensetzt. Hierbei wird der Patient intensiv durch Ergotherapeuten begleitet, meist in einer 1:1-Situation. Das multidisziplinäre Team einschließlich des Schmerztherapeuten sorgt für die optimale Betreuung des Patienten, was insbesondere im Hin- blick auf Kontextfaktoren und Schmerzen ein entscheidender Vorteil ist.

Am Ende der Maßnahme erfolgt die Abschlussuntersuchung wieder durch das interdisziplinäre Team und mit Beteiligung des Rehabilitationsmanagers des Kostenträgers. Hierbei wird ermittelt, ob der Patient noch persönliche Schwächen durch den Unfall hat und in welchem Maße es gelungen ist, Defizite und Einschränkungen durch die Rehabilitationsmaßnahme zu beseitigen. Im Team werden Zukunftsperspektiven gemeinsam mit dem Patienten entwickelt, damit dieser den Einstieg zurück in seinen alten Beruf schafft.

Die medizinisch-beruflich orientierte Rehabilitation wird wissenschaftlich begleitet und evaluiert. Alle Patienten werden bei Abschluss der Therapiemaßnahme und 6 Monate nach Therapieende untersucht. Hierbei soll die langfristige Wirkung der MBO-Rehabilitation belegt werden. Die durch die Nachuntersuchung gewonnenen Ergebnisse fließen kontinuierlich in die Verbesserung des Leistungsangebots durch die interdisziplinäre Arbeitsgruppe ein ( $\bullet$ Abb.3).

\section{In der BG-Unfallklinik Ludwigshafen abgebildete Berufsbilder}

Durch Umbaumaßnahmen für über 200.000 EUR wurden die Voraussetzungen geschaffen, um ein breites Spektrum an Arbeitsplätzen (- Abb.4) in folgenden Bereichen abzubilden:

\section{Kraftfahrer}

Tätigkeiten, Funktionen. Ein- und Aussteigen, Fahren, Lenken, Reifenmontage, Be- und Entladen, Fixieren von Spanngurten, Schließen der Ladeklappe, Umgang mit Lasten

Geräte. BTE-Simulator (BTE: Baltimore, Therapeutic Equipment), Fahrschule mit LKW, Simulation Reifenmontage, Simulation Lastenhandhabung z. B. mit Rollcontainer, LKW-Laderampe, Hubwagen, verschiedene Spanngurtfixierungen

\section{Maschinen/Anlagen}

Tätigkeiten, Funktionen. Beispielsweise Fräsen, Bohren, Schneiden von Rohrgewinden, Trennen von Blechen/Rohren/Profilen, Formen von Bauteilen, Einbau von Rohrleitungsteilen und Armaturen, Herstellen von Verbindungen mit Flansch oder Schraubarmaturen, Sichern und Transportieren von Bauteilen, Bedie- 

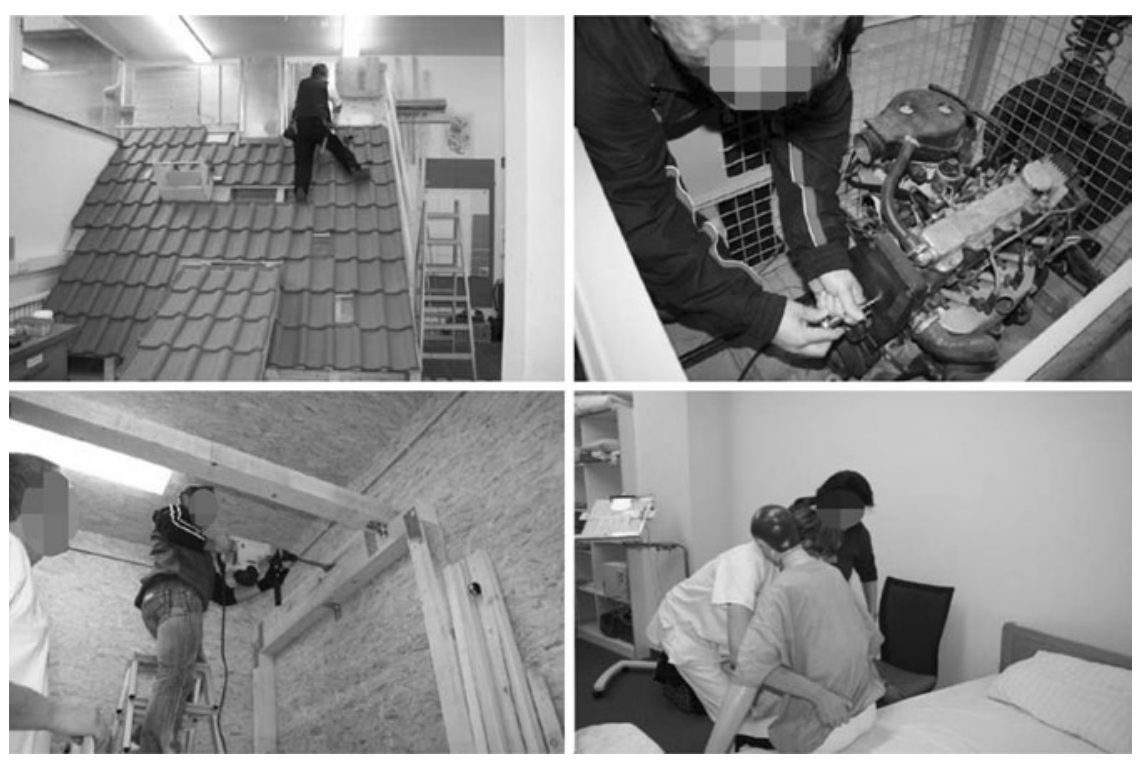

Abb. $4 \Delta$ Ausschnitt aus dem Leistungsspektrum der MBO-Rehabilitation an der BG-Unfallklinik Ludwigshafen

nung von Transport- und Hebemaschinen, Aufbau von Arbeitsgerüsten

Geräte. Metallraum, um die genannten Tätigkeiten zu erproben; BTE-Maschinensimulation, Drehbank, Gerüst, Lastenhandhabung

\section{Kfz, Elektrotechnik}

Tätigkeiten, Funktionen. Überkopfarbeiten, Zwangshaltungen, Radwechseln, Fein- und Präzisionsarbeit

Geräte. PKW, zerlegbarer PKW, Hebebühne oder Graben, Gitterkasten zur Erprobung und zum Training von Tätigkeiten in Zwangshaltung, Werkzeug zur Testung der Feinmotorik, verschiedene Tätigkeiten an Überkopfarbeitsplätzen

Büro

Tätigkeiten, Funktionen. Schreiben, Bedienen eines PC, Sitzen, Telefonieren, Arbeit unter Zeitdruck, Aktenverwaltung

Geräte. Computerarbeitsplatz mit Möglichkeit der Umsetzung der Sitz- und Stehdynamik, verschiedene ergonomische Bürostühle und Tastaturen, Wandregal
Labor, Chemie

Tätigkeiten, Funktionen. Feinmotorik, Hand-/Fingerkoordination

Geräte. Feinmotorische Übungsgeräte, Laborgeräte (Reagenzgläser, Pipetten)

Lager

Tätigkeiten, Funktionen. Lastenhandhabung unterschiedlicher Art (Kisten, Container, Pakete, Paletten, andere Verwahrungssysteme) unter Benutzung von Leitern, Hubwagen, Gabelstapler oder anderen Transport- und Hilfssystemen

Geräte. Regale, Gewichte, Leiter, Hubwagen und andere Transportmittel

\section{Küche, Gastronomie}

Tätigkeiten, Funktionen. Tragen, Balance, Feinmotorik

Tätigkeiten, Funktionen. Tragen, Schleppen, Knien, Klettern, Mauern, Steine setzen, Pflastern, Kabelrollen bewegen, Arbeit mit erhöhter Unfallgefahr, Zwangshaltung, Arbeit auf Gerüsten

Geräte. Hubwagen, Holzbohlen, Schubkarre, Zementsäcke, Gerüst, Pflasterbereich, überdachter Arbeitsplatz, Holzraum, Dachschräge

Garten-, Landschaftsbau, Forstwirtschaft

Tätigkeiten, Funktionen. Tätigkeitsfeld sehr variabel

Geräte. Individueller Einsatz der Geräte nach Tätigkeitsanalyse

\section{Pflege, Krankenversorgung}

Tätigkeiten, Funktionen. Umbetten, Feinmotorik, Schreiben

Geräte. Übungsbett, BTE-Simulator, Kooperation mit Pflegedienst des Hauses, MTT (medizinische Trainingstherapie)
Räume, Geräte. Therapieküche, verschiedene Transportsysteme, Simulation der Kerntätigkeit nach Angaben des Patienten oder der Arbeitsplatzbeschreibung

\section{Verkauf, Kasse}

Tätigkeiten, Funktionen. Stehen, Sitzen, Handhabung von Gewichten unterschiedlicher Form, Größe und Beschaffenheit, Feinmotorik

Geräte. Kassenarbeitsplatz mit Objekten nach individueller Situation, BTE-Simulation, feinmotorische Arbeitsaufgaben aus den ergotherapeutischen Therapieangeboten

\section{Weitere Bereiche}

Die MBO-Rehabilitation kann auch in den Bereichen Feuerwehr, Sicherheitsdienst und Spitzensport durchgeführt werden.

\section{Individuelle Betreuung}

Selbstverständlich erfolgt die Auswahl der Maßnahmen und der Übungs- und Trainingsgeräte grundsätzlich nach den individuellen Problemen und Fähigkeits- 


\section{Psychische, berufliche und soziale Aspekte}

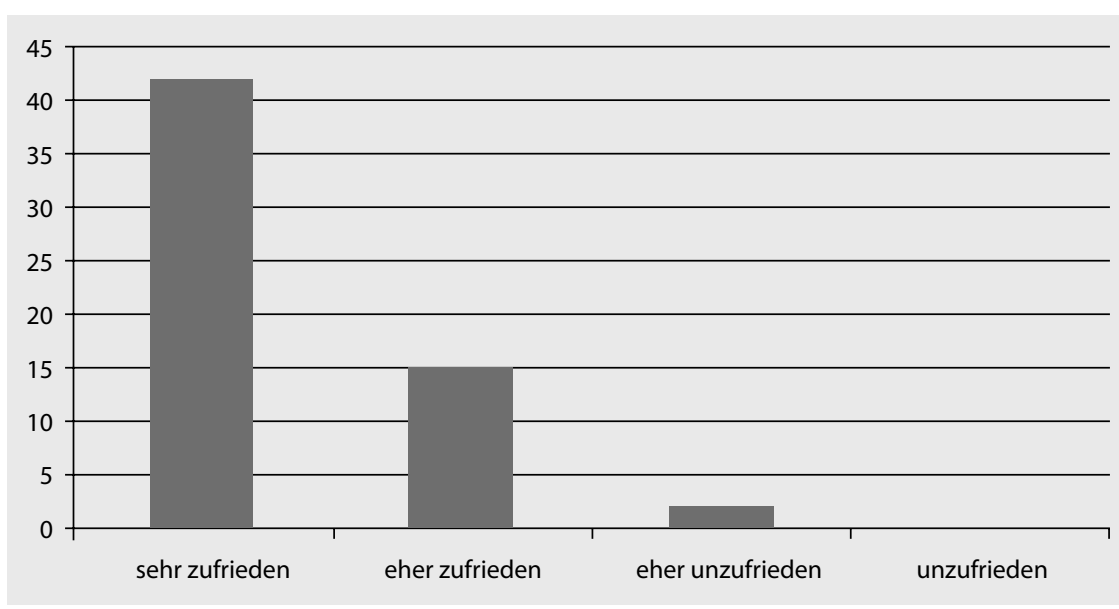

Abb. $5 \Delta$ Patientenzufriedenheit bei Entlassung aus der MBO-Rehabilitationsmaßnahme an der BGUnfallklinik Ludwigshafen

störungen der Patienten und nach der Arbeitsplatzbeschreibung.

Entscheidender Vorteil ist, dass die Therapie direkt in der Klinik stattfindet und nicht in Firmen in der Umgebung. Dabei werden die Patienten durch Fachpersonal betreut - im Unterschied zu anderen Maßnahmen, bei denen die Patienten nach einer kurzen Anleitung durch den Therapeuten ohne Betreuung in Firmen arbeiten.

\section{Indikationen zur MBO-Rehabilitation}

Das Einzugsgebiet der Patienten für die medizinisch-beruflich orientierte Rehabilitation umfasst Deutschland. Grundsätzlich kommt dieses neue Rehabilitationsverfahren für folgende Personen in Betracht:

- Patienten, bei denen die individuelle Leistungsfähigkeit auch nach den üblichen Standardmaßnahmen nicht den beruflichen Leistungsanforderungen entspricht,

- Patienten, bei denen die beruflichen Anforderungen besondere Fähigkeiten z. B. in Bezug auf Geschicklichkeit, Feinmotorik oder Reaktionsfähigkeit erfordern, die in den üblichen Standardmaßnahmen nicht hinreichend geübt und getestet werden können, ebenso Patienten, die beruflich spezifischen körperlichen Belastungen ausgesetzt sind,

- Patienten nach einem bereits gescheiterten beruflichen Eingliederungsver- such, die aber keiner Standardrehabilitation bedürfen, sondern lediglich berufsspezifisch üben müssen,

- Patienten, bei denen am Arbeitsplatz keine Belastungserprobung möglich ist,

- Patienten, bei denen besondere Kontextfaktoren eine Rolle spielen. Hierzu gehören u. a. psychosoziale Probleme im Beruf (Angst vor der Arbeitsstätte, Angst vor Schmerz, mangelndes Gefühl der Selbsteffektivität, Probleme mit Motivation, Kommunikation und Interaktion).

\section{Erste Ergebnisse}

Vom 01.01.2009 bis zum 30.09.2011 nahmen insgesamt über 300 Patienten an der medizinisch-beruflich orientierten Rehabilitation teil, bei 232 der nachuntersuchten Patienten wurden die Ergebnisse bereits ausgewertet.

Bei den nachuntersuchten Patienten zeigte sich, dass die veranschlagte Therapiedauer von 4 Wochen in den meisten Fällen ausreichend war, nur wenige $\mathrm{Pa}$ tienten mussten die Rehabilitationsmaßnahme verlängern.

63\% der Patienten waren nach der Entlassung aus der MBO-Rehabilitation sofort arbeitsfähig oder konnten mit einer verkürzten Arbeits- und Belastungserprobung in ihre früheren Tätigkeiten integriert werden. Nur in Ausnahmefällen mussten Patienten berentet werden. Angesichts der Schwere der Verletzungen bzw. der z. T. besonderen Kontextfaktoren ist das ein sehr gutes Ergebnis.

Die Patienten erlebten und bewerteten die medizinisch-beruflich orientierte Rehabilitation als sehr positiv, insbesondere den Verlauf des stationären Aufenthalts und das spezifische Therapieangebot in Abstimmung auf die berufliche Leistungsanforderung ( $\mathbf{A b b}$. 5). Durch eine schriftliche Patientenbefragung sollen diese Eindrücke in den kommenden Monaten noch validiert werden. Die Patienten fühlten sich durch die Einbeziehung in die Visiten und in das gemeinsame Erarbeiten von Therapiezielen ernst genommen.

Der persönliche und direkte Bezug zum Therapeuten fand seitens der Patienten großen Zuspruch.

\section{Fazit für die Praxis}

Die Ergebnisse der medizinisch-beruflich orientierten Rehabilitation zeigen einen äußerst positiven Trend bezüglich der Wiedereingliederung des Unfallverletzten in das Arbeitsleben trotz schwerer Verletzungsfolgen oder bereits gescheiteter ABE-Maßnahme (ABE: Arbeitsbelastungserprobung). Kein bisher etabliertes Rehabilitationsverfahren erreicht eine derart hohe Erfolgsquote hinsichtlich der Rückkehr an den alten Arbeitsplatz. Bisher standen alle Patienten diesem neuen Verfahren positiv gegenüber und arbeiteten extrem motiviert mit. Damit ist die medizinisch-beruflich orientierte Rehabilitation ein zweifach kundenorientiertes Rehabilitationsverfahren mit großer Zukunftsperspektive.

\section{Korrespondenzadresse}

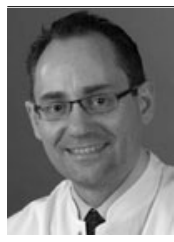

Dr. H. Kohler

Abteilung für Berufsgenossenschaftliche Rehabilitation und Heilverfahrenssteuerung, BG-Unfallklinik Ludwigshafen, Ludwig-Guttmann-Straße 13, 67071 Ludwigshafen hkohler@bgu-ludwigshafen.de 
Interessenkonflikt. Der korrespondierende Autor gibt an, dass kein Interessenkonflikt besteht.

The supplement containing this article is not sponsored by industry.

\section{Literatur}

1. Bundesarbeitsgemeinschaft für Rehabilitation (1999) Positionspapier der Bundesarbeits-Gemeinschaft für Rehabilitation zur Weiterentwicklung der medizinischen, beruflichen und sozialen Rehabilitation in der 14. Legislaturperiode - beschlossen am 15. Dezember 1998. Bundesarbeitsgemeinschaft für Rehabilitation, Frankfurt, Main

2. Bürger W (2006) Entwicklungsstand der berufsbezogenen Angebote in der medizinischen Rehabilitation. In: Müller-Fahrnow W, Hansmeier T, Karoff M (Hrsg) Wissenschaftliche Grundlagen der medizinisch-beruflich orientierten Rehabilitation. Pabst, Lengerich, S 47-55

3. Grüber K (2011) Die UN-Konvention über die Rechte von Menschen mit Behinderungen. DGUV Forum $5 / 11$

4. Harth A, Germann G, Jester A (2006) Evaluating the effectiveness of a patient-oriented-programme in hand rehabilitation. J Hand Surg Eur 33:E6

5. Hillert A, Müller-Fahrnow W, Radoschewski FM (2009) Medizinisch-beruflich orientierte Rehabilitation. Grundlagen und klinische Praxis.Deutscher Ärzteverlag, Köln

6. Krüger H, Schöttler B, Kiesel J (2006) Erste Erfahrung mit einem $\mathrm{MBO}$-REHA-Angebot bei onkologischen Patienten. In: Müller-Fahrnow W, Hansmeier T, Karoff M (Hrsg) Wissenschaftliche Grundlagen der medizinisch-beruflich orientierten Rehabilitation. Pabst, Lengerich

7. Müller-Fahrnow W, Knörzer J, Muraitis A et al (2005) Ergebnisevaluation der MBO-REHA Rehabilitation von MSK Patienten. 14. Rehabilitationswissenschaftliches Kolloquium. DRV Schriften 59

8. Müller-Fahrnow T, Hansmeier M, Karoff M (2006) Wissenschaftliche Grundlagen der medizinischberuflich-orientierten Rehabilitation. Pabst, Lengerich

9. Plassmann R, Färber K (1995) Rentenentwicklung bei psychosomatisch Kranken. Rehabilitation 34:23-27

10. Schliehe F, Egner U, Streibelt (2011) MBOR - ein Prozessmodell in der medizinischen Rehabilitation. Die Rehabilitation, Bd 50. Thieme, Stuttgart New York

11. Streibelt M, Hansmeier T, Muller-Fahrnow W (2006) Effekte berufsbezogener Behandlungselemente in der orthopädischen Rehabilitation der Rentenversicherung. Ergebnisse einer randomisierten Verlaufsstudie. Rehabilitation 45:161-171

12. Zielke M, Carls W, Evertz P et al (2006) Zur Konfundierung zwischen den Qualitätsstandards einer Rehabilitationsklinik und den Qualitätsbefragung durch Patienten. Praxis Verhaltenstherapie 71:311 